\title{
An Extension of MAC Protocol for Mobility Detection and Efficient Data Transmission
}

\author{
Ankur V. Gondha \\ Student: (M.Tech. - E\&C) \\ Electronics and Communication Engineering \\ Department \\ School of Engineering \\ RK University \\ Rajkot, India.
}

\author{
Arjav A. Bavarva \\ Assistant Professor \\ Department of Electronics and Communication \\ Engineering \\ School of Engineering \\ RK University \\ Rajkot, India.
}

\begin{abstract}
This paper introduces an extension of MAC protocol for mobility detection of nodes and efficient data transmission in mobile, multihop wireless sensor network. Here for mobility detection, receiving power of the node is consider by the transmitting node. If gradual degradation in communication link does due to degradation in receiving power, it initiates the switchover of link via the relay node for efficient data transmission. Our present implementation is done by extending the energy efficient IEEE 802.11 MAC protocol because most commonly used standard is IEEE 802.11 in sensor networks consist of small, inexpensive, resource constrained devices that communicate wirelessly in a multihop network. Here the paper shows the performance of extended MAC protocol in terms of average throughput, packet loss and average delay by varied the parameters like mobility of node and size of the packet.
\end{abstract}

\section{General Terms}

Mobility, Switchover, MAC protocol, Wireless sensor network

\section{Keywords}

MAC Protocol, Mobility, Wireless Sensor Network, Packets, Switchover, Relay node, Throughput

\section{INTRODUCTION}

The wireless sensor network is an infrastructure less network that consist of small sensors nodes scattered in the sensing environment and one or more sink node(s).In some of the applications like continuous or periodic monitoring of the patients to track their activities or to measure physiological parameter like blood pressure of patients by moving doctor in hospital. In such applications, sensor nodes are deployed on mobile humans (patients \& doctors), to track activities. The common consideration of such applications is pedestrian mobility, i.e., the movement of nodes (doctors) is held in a limited area such as a floor or a building. Due to their mobility in such application needs the efficient data transmission without link breakage and data loss. To avoid such situations network needs dominant and dynamic reconfiguration.

In this paper we introduce the extension of energy efficient IEEE 802.11 MAC protocol for efficient data transmission by detecting the mobility and switchover of communication link to the relay node in time. This concept of mobility detection and switchover of packet is considered from the MAMAC protocol.
To avoid packet / message collisions, a media access control (MAC) protocol is needed.Means MAC protocol controls when to send and receive distinguished packet between nodes in a network. Manipulating the operation of a MAC protocol can give its effect in terms of energy. All MAC protocols for wireless network manage the usage of the radio link interface for efficient utilization of the shared bandwidth. MAC protocols designed for wireless sensor network have an additional goal of managing radio activity to conserve energy. Thus, while traditional MAC protocols must balance throughput, delay, and fairness concerns.

Without considering mobility of sensor nodes, delay is produced due to gradual degradation in communication link quality and ultimately link break down occurs [1]. To maintain efficient communication in presence of mobility of nodes on either side in wireless sensor network requires efficient Mobility Aware MAC protocols like MS-MAC, MMAC, M_TDMA, MA-MAC, MCMAC, MobiSense, etc are required.

\section{RELATED WORK}

In the mobile environment, wireless sensor network should be efficient enough to detect the mobility of sensor nodes or sink nodes to avoid the energy wastage by avoiding the retransmission. On the basis of that mobility can be classified in three types I) Node mobility, II) Sink mobility and III) Event mobility.

The sink (Doctor) moves randomly or along a deterministic path in hospital. The goal is to reduce packet loss and to redistribute the energy consumption and increased throughput in the network. In the 802.11-standard stations perform active or passive scanning across the full ISM (Industrial, Scientific, and Medical) band to discover nearby access points. The distance measurement of sensor node can be done through RSSI, GPS, RFID, SNR or Ultrasound, etc. Among all these here we have selected RSSI for detection of mobility of node in terms of current position of node. Because it is comparatively inexpensive technique to measures the position of mobile node [2]. After detection of mobility, switchover of ongoing transmission between transmitting sensor node and mobile sink node considers in time to avoid the link breakage and retransmission. For that two distance threshold levels are selected. After detection of first threshold level by transmitting node, it initiates the switchover process to relay (neighboring node of transmitting node in the coverage region of both transmitting and receiving node) node. While second distance threshold level sets an upper limit to complete the 
switchover process. Means switchover process must be completed in between two distance threshold level. If switchover process is not done in time link breakage occurs due to gradual degradation in communication link quality and such situation is unavoidable.

\section{CONCEPT}

Here proposed extended MAC protocol completes the detection of mobility in ongoing transmission. In such situation following solution can be considered to avoid the link breakage of ongoing transmission and retransmission [1].

I) The node needs to complete transmission of all the data before a link breaks, and hence, there is no need to deal with mobility.

II) The node needs to adapt dynamic transmission rate, so it transmits data packets at higher rate before link breaks.

III) The node needs to initiate and complete switchover before ongoing transmission link breaks.

To take decision from above requires the link layer to control on information that comes from the application, network, and physical layers. The application/network layer provides information about the size of the data packet. The physical layer provides information about the different transmission rates that can be supported by the radio.

An extended MAC protocol detects the mobility of receiving node by continuous measurement of received signal strength of ACK packets. After detection of mobility it sets two thresholds to complete the switchover. In switchover process selection of neighbor node is done parallel by embedding the request packets in ongoing transmission. For that ongoing unicast transmission is switchover to broadcast in parallel with ongoing transmission. After reception of request packets to the neighbor nodes it replies o the request transmitting node. Among all neighbor node nearest node that reply first is selects as a relay node in multihop communication.

During switchover process and after successful switchover packet drop is observed. This decreases the average throughput of the network. Such a packet loss is decreased by decreasing the size of the data packet without changing the transmission rate. This conceptually also avoids the delay in transmission by decreasing the size of packet.

\subsection{Mobility and Mobility Model}

In the mobile environment, wireless sensor network should be efficient enough to detect the mobility of sensor nodes or sink nodes or event to avoid the energy wastage. On that basis mobility is be classified following manner [3].
I) Node mobility
II) Sink mobility
III) Event mobility

For mobility estimation in different application of mobile wireless sensor network, knowledge of mobility pattern requires. Mobility pattern is a movement of the real time subjects or objects. And such a movement is considered as a realistic mobility pattern. Realistic mobility patterns can be classified as pedestrian mobility, vehicular mobility, aerial mobility, dynamic medium, robotic movements, and outer space motion [4]. Among all these here we have considered the pedestrian mobility pattern because it best suits for the considered application of wireless sensor network. In pedestrian mobility pattern slow movement of mobile node (Doctor) is considered as 0.5 meter/second while fast movement of mobile node (Doctor) is considered as 1.5 meter/second [1].

\subsection{Mobility Estimation}

The extended MAC protocol's implementation is done using network simulator 2.35. In that implementation we have considered Two Ray Ground Reflection mode and network interface type Wireless Physical for the calculation of received power using equation (1). According to the Two Ray Ground Reflection model received power can be measured using following equation [5].

$$
\operatorname{Pr}=\frac{\mathrm{Pt} * \mathrm{Gt} * \mathrm{Gr} * \mathrm{ht}^{2} * \mathrm{hr}^{2}}{d^{4} * L}
$$

$\operatorname{Pr}=$ Received power in $\mathrm{dBm}$.

$\mathrm{Pt}=$ Transmitted power in $\mathrm{dBm}$.

$\mathrm{Gt}=$ Transmitting antenna Gain in $\mathrm{dBm}$.

$\mathrm{Gr}=$ Receiving antenna Gain in $\mathrm{dBm}$.

$h t=$ Height of transmitting antenna in meters.

$\mathrm{hr}=$ Height of receiving antenna in meters.

$\mathrm{L}=$ Path loss exponent in $\mathrm{dBm}$.

By using equation we have measured the RSSI means received power of the mobile sink /node. After measurement, following logic was implemented for mobility detection and for handover to the neighbour node.

\section{RX_Thresh1 $<$ Pa $<$ RX_Thresh2}

\author{
RX_Thresh1 $=$ Initial distance threshold level. \\ RX_Thresh2 $=$ Upper distance threshold level. \\ $\mathrm{Pa}=$ Node presently receiving packets $\mathrm{t}$ this \\ power level.
}

We set the value of initial threshold level RX_Thresh1 $=30 \mathrm{dBm}$ and upper limit of the threshold level RX_Thresh2 = 80dBm.According to above logic, if static transmitting node receiving packets from mobile node at Pa power level and it is greater than RX_Thresh1 then it considers mobile receiving node is moving away from the static transmitting node. While after detection of mobility handover process does before it reaches up to the RX_Thresh2.

\section{IMPLEMENTATION}

For the implementation of extended MAC protocol we have implemented scenario as shown in the Fig. 1. As shown in the Fig. 1 we have created one static sender node TX, receiving node RX and two neighbour nodes N1, N2.In phase-1 al three nodes accepting TX node are in the range of TX node and static. Here coverage region of TX node is shown as a blue circle. In second phase RX node is moving away from the TX node while neighbours N1, N2 are static. In second phase TX node detects the mobility of node RX by continuous monitor the receive power level of the acknowledgement packets. When receive power level crosses the initial threshold level $(30 \mathrm{dBm})$ in parallel to data transmission TX starts the broadcasts the request packets to the neighbour nodes. 


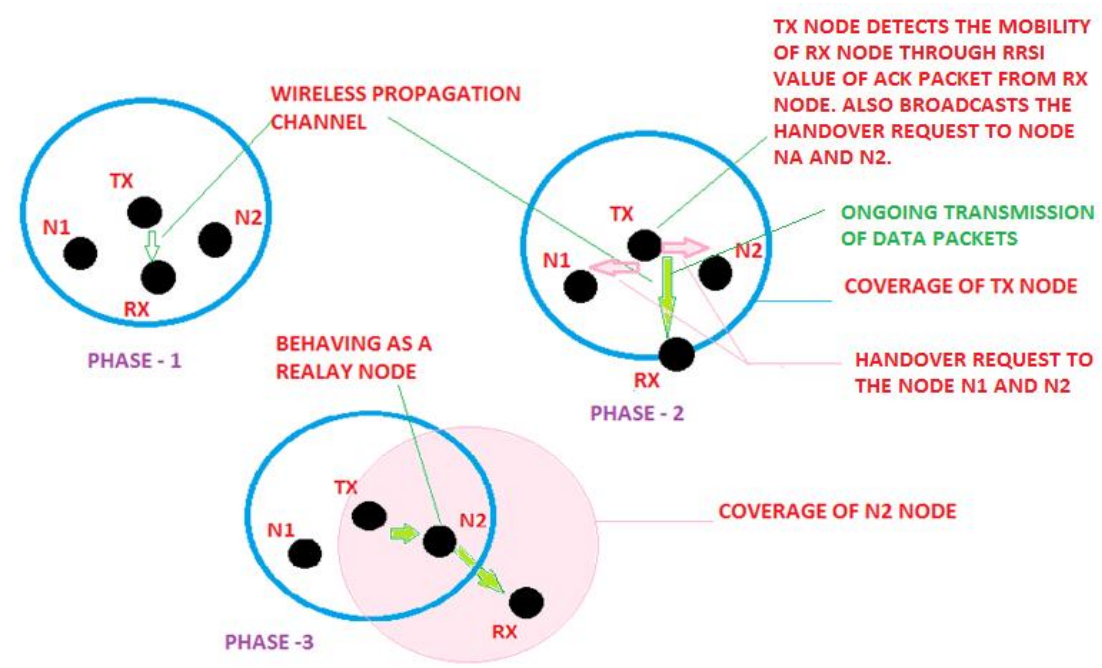

Fig1: Implemented Wireless Scenario in Network Simulator (NS2)

This process of request packet transmission to the neighbour node and also the switch over to the relay (neighbour) node should be done before received power level reaches to the upper limit of distance threshold level $(80 \mathrm{dBm})$.

In third phase neighbour node N2 reply first to the TX node's request and behaves as a relay node and successful data transmission achieved.

Here in this implementation we have selected two different mobility 0.5 meters/second and 1.5 meters/second [1]. In both the case we have selected the UDP connection in between nodes and CBR packets having size of 1024 bytes and data rate of $600 \mathrm{~Kb} /$ second. In this implementation packets drop is observed during and after switchover process. Ultimately this decreases the average throughput of the network. To increase the average throughput and to decrease the packet loss size of the data packets is reduced to 512 bytes with the same data rate of $600 \mathrm{~Kb} / \mathrm{second}$.

\section{SIMULATION RESULTS}

We have implemented the wireless scenario shown in Fig. 1 in network simulator we generated the trace file of packet loss, throughput and delay for both the case of mobility 0.5 meters/second and 1.5 meters/second with different packet size but with the same data rate.

\subsection{Packet Loss}

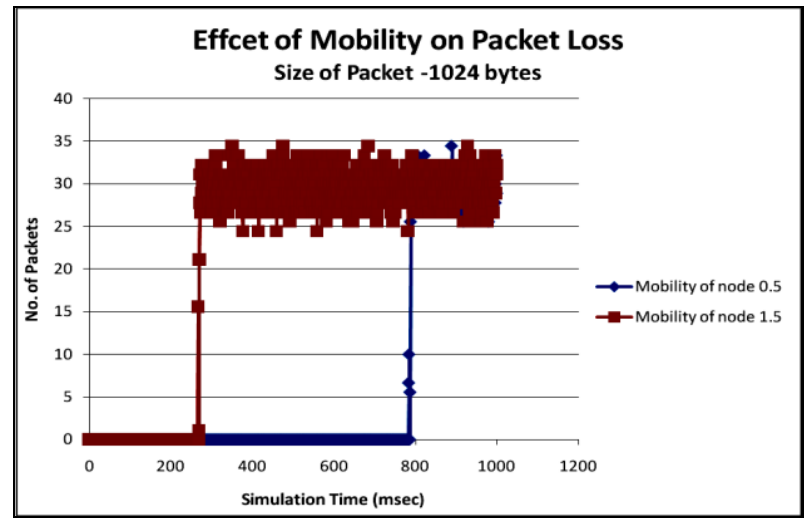

Fig. 2: Packet loss when packet size is 1024 bytes
Here we have implemented the scenario shown in Fig. 1 using network simulator 2.35 with the two different mobility 0.5 meters/second and 1.5 meters/second with UDP connection between nodes. In first case same CBR packet size 1024 bytes with $600 \mathrm{~Kb} /$ seconds data rate packet loss is observed as shown in the Fig. 2.

As shown in Fig. 2 maximum loss of packets is 35 in both the case of mobility. But in case of 0.5 meters/second mobility packets drop is started at $800 \mathrm{msec}$ simulation time. While in case of 1.5 meters/sec mobility packets drops is started in between 200 to $400 \mathrm{msec}$ simulation time. In both cases simulation ended at $1000 \mathrm{msec}$.

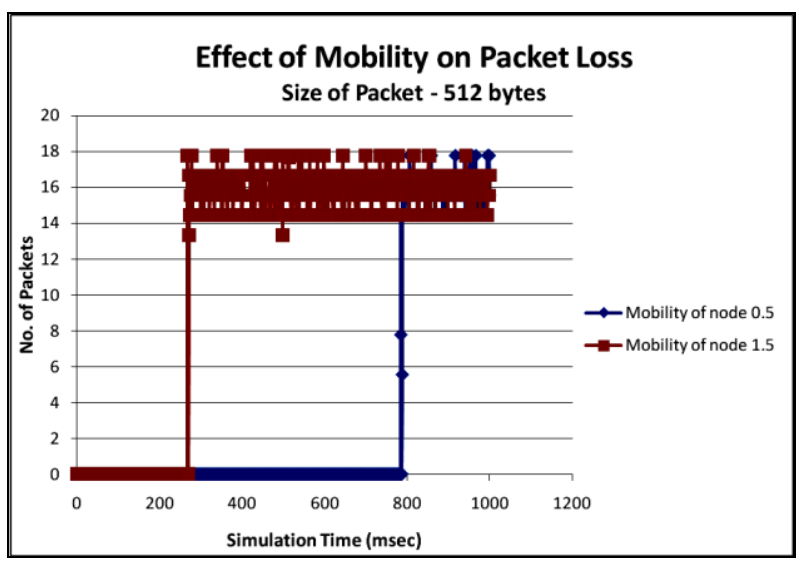

Fig. 3: Packet loss when packet size is 512 bytes

As shoen in the Fig. 3 maximum loss of packets is 18 in both the cases of mobility. But in case of 0.5 meters/second mobility packets drop is started at 800 msec simulation time. While in case of 1.5 meters/sec mobility packets drops is started in between 200 to $400 \mathrm{msec}$ simulation time. In both cases simulation ended at $1000 \mathrm{msec}$.

\subsection{Average Throughput}

Throughput of the network is actual capacity of network to carry the data packets and bandwidth is the maximum capacity of network to carry tha data packets. So, Throughput is always less tha bandwidth in practical conditions. 
Here we have implemented the same scenario with both case of mobility by kept the CBR packet size 1024 bytes and 512 bytes with same data rate $600 \mathrm{~Kb} /$ seconds and observed the different throughput in Fig. 4 and Fig. 5 respectively.

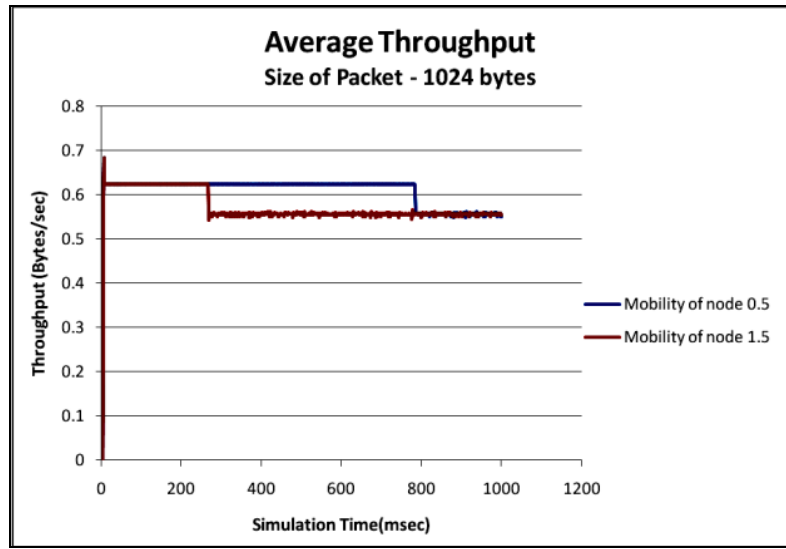

Fig. 4: Throughput of network when packet size is 1024 bytes

As shown in the Fig. 4 average throughput of network is stable upto $800 \mathrm{msec}$ simulation time and then decreses when mobility is 0.5 meters/second. While mobility is 1.5 meters/second, average throghput of network is stable up to near about $250 \mathrm{msec}$ simulation time and then started to decrease.

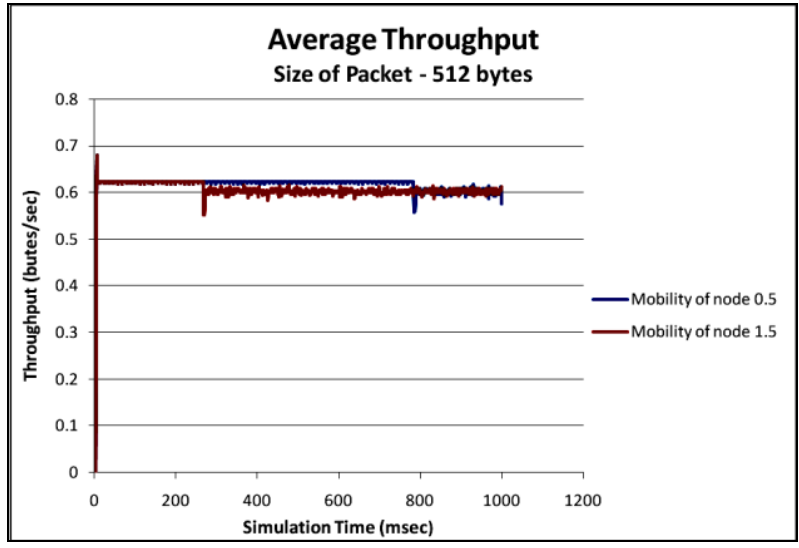

Fig. 5: Throughput of network when packet size is 512 bytes

As shown in the Fig. 5 average throughput of network is stable upto 800 msec simulation time and then decreses when mobility is 0.5 meters/second. While mobility is 1.5 meters/second, average throghput of network is stable up to near about $250 \mathrm{msec}$ simulation time and then started to decrease. Here in Fig. 5 decrement in second case of mobility 1.5 metrs/second is less compared to Fig. 4. This shows that Throughput is improved.

\subsection{Average Delay}

When any event is detected by sensor node which is mobile in wireless sensor network it has to be reached in time to the sink node in time otherwise it will not be considered as useful information. So, such lack of time in event detection application which is mobile is called delay. Here we observed the delay pattern with both case of mobility and 1024 bytes of packet size and 512 bytes in Fig. 6 and Fig. 7 respectively.

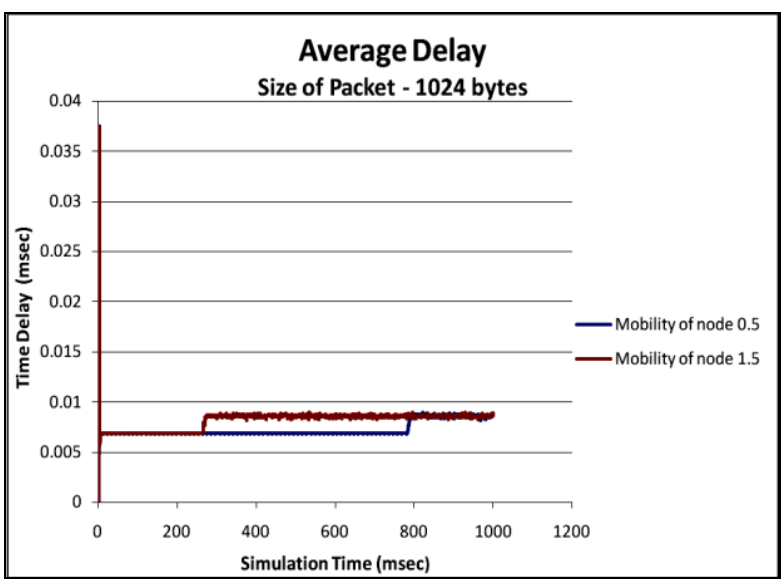

Fig. 6: Average Delay when packet size is 1024 bytes

As shown in the Fig. 6 average delay is observed more in case 1.5 meters/ sec mobility compared to 0.5 meters/second mobility.

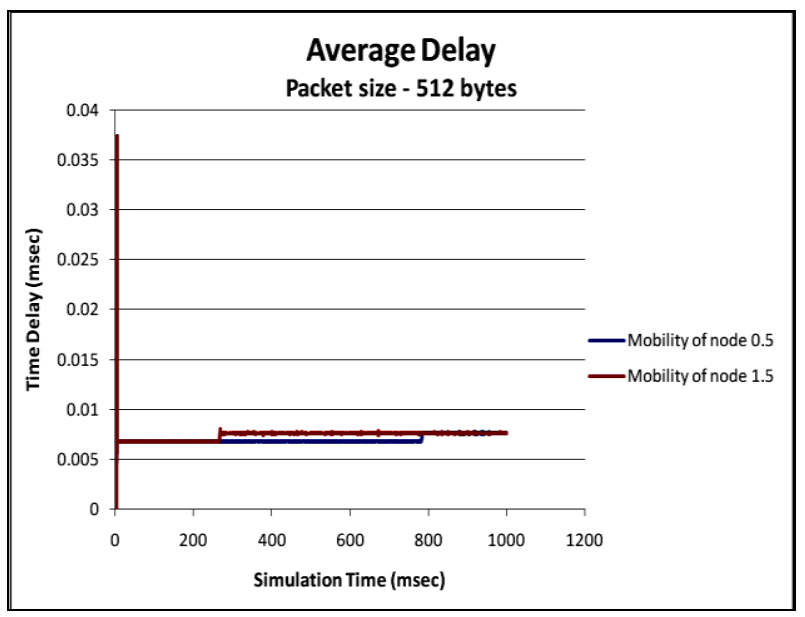

Fig. 7: Average Delay when packet packet size is 512 bytes

As shown in the Fig. 7 average delay is observed still more in case 1.5 meters/ sec mobility compared to 0.5 meters/second mobility. But in comparison of 1024 packet size delay is less observed in case of mobility 1.5 meters/second.

\subsection{Comparative analysis}

By using the AWK script to calculate the sent packet, received packet, ratio of received to send packet and forwarded packet by relay node in this implementation in network simulator we found the results shown in Table 1 and Table 2 for both the case of node mobility.

Table 1. Comparative analysis when node mobility is 0.5 metres / Second

\begin{tabular}{|c|c|c|c|c|}
\hline \multicolumn{5}{|c|}{ Mobility of node $\mathbf{0 . 5}$ meters/second } \\
\hline $\begin{array}{c}\text { Size of } \\
\text { Packet }\end{array}$ & $\begin{array}{c}\text { Send } \\
\text { Line } \\
(\mathbf{s})\end{array}$ & $\begin{array}{c}\text { Receive } \\
\text { Line (r) }\end{array}$ & r/s & $\begin{array}{c}\text { Forward } \\
\text { Line (f) }\end{array}$ \\
\hline 512 bytes & 146280 & 142623 & 0.9750 & 28215 \\
\hline 1024 bytes & 146280 & 139644 & 0.9546 & 25240 \\
\hline
\end{tabular}


As shown in Table 1 packet loss is decreased when size of packet is 512 bytes.

Table 2. Comparative analysis when node mobility is $\mathbf{1 . 5}$ metres / Second

\begin{tabular}{|c|c|c|c|c|}
\hline \multicolumn{5}{|c|}{ Mobility of node 1.5 meters/second } \\
\hline $\begin{array}{c}\text { Size of } \\
\text { Packet }\end{array}$ & $\begin{array}{c}\text { Send } \\
\text { Line } \\
\text { (s) }\end{array}$ & $\begin{array}{c}\text { Receive } \\
\text { Line (r) }\end{array}$ & r/s & $\begin{array}{c}\text { Forward } \\
\text { Line (f) }\end{array}$ \\
\hline 512 bytes & 146280 & 134396 & 0.9188 & 95750 \\
\hline 1024 bytes & 146280 & 124229 & 0.8493 & 85584 \\
\hline
\end{tabular}

As shown in the Table 2 packet loss is decreased when size of packet is 512 bytes.

\section{CONCLUSION}

In this paper extended MAC protocol detects the mobility of node by continuous measurement of received power and successful switchover of transmission link to the relay node is done by setting the two distance threshold levels (30dBm and $80 \mathrm{dBm}$ ) in time without affecting the efficiency of network. From this implementation it is observed size of data packets matters whenever average throughput and packet loss are important factors of in case of mobile wireless sensor application. Here whenever packet size is 512 bytes average throughput was increased up to 0.0204 compared to 1024 bytes packet size in case of node mobility 0.5 meters/second. While in case of node mobility 1.5 meters/ second average throughput was increased up to 0.0695 when packet size is 512 bytes compared to 1024 bytes packet size.

\section{ACKNOWLEDGEMENTS}

I thank, Asst. Prof. A. A. Bavarva, School of Engineering, R.K University, Rajkot, Gujarat, India for his valuable guidance, constant encouragements, over willing help and sustained interest right from the selection of my topic up to the final shaping.

\section{REFERENCES}

[1] W. Dargie, "A medium access control protocol that supports a seamless switchover in wireless sensor network", Journal of Network and Computer Applications 35 (2012) 778 - 786.

[2] Q. Dong, W. Dargie, "A Survey on Mobility \& Mobility Aware MAC Protocols in Wireless Sensor
Network", IEEE COMMUNICATION \& TUTORIALS, Vol.15, NO.1, FIRST QUARTER, pp. 88-100, 2013

[3] H. Karl and A. Willig, "Protocols and Architectures for Wireless Sensor Networks", John Wiley \& Sons, 2005.

[4] C. Schindelhauer, "Mobility in Wireless Networks", J. Wiedermann et al. (Eds.): SOFSEM 2006, LNCS 3831, pp. 100-116, Springer-Verlag Berlin Heidelberg, 2006.

[5] T. S. Rappaport, "Wireless Communications- Principles and Practice", Dorling Kindersely (India), 2010.

[6] U. C. Berkeley, K. Fall, K. Varadhan "The ns Manual (formerly ns Notesand Documentation)", [Online], Available: http://www.isi.edu/nsnam/ns/ns-ocumentation

[7] M. Nabi, M. Blagojevic, M. Geilen, T. Basten, and T. Hendriks, "MCMAC: An optimized medium access control protocol for mobile clusters in wireless sensor networks," 7th Annual IEEE Communications Society Conference on Sensor Mesh and Ad Hoc Communications and Networks (SECON), pp. 1-9, June 2010.

[8] M. Ali, T. Suleman, and Z. A. Uzmi, "MMAC: A Mobility- Adaptive, Collision-Free MAC Protocol for Wireless Sensor Networks", IEEE 2005, pp. 401-407.

[9] A. Gonga, O. Landsiedel, and M. Johansson, "MobiSense: Power efficient micro-mobility in wireless sensor networks," Proc. 7th IEEE International Conference on Distributed Computing in Sensor Systems, June 2011.

[10] H. Pham and S. Jha, "An adaptive mobility-aware mac protocol for sensor networks (ms-mac)," IEEE International Conference on Mobile Ad-hoc and Sensor Systems, pp. 558 - 560, October 2004

[11] W. Ye, J. Heidemann, and D. Estrin, "An energyefficient mac protocol for wireless sensor networks," in Proc. IEEE INFOCOM, New York, NY, pp. 1567-1576, June 2002

[12] M. Srbinovska, V. Dimcev, C. Gavrovski and Z. Kokolanski, "Localization Techniques in Wireless Sensor Networks using Measurement of Received Signa Strength Indicator" Electronics, Vol. 15, No. 1, June 2011. 